INFORME BREVE

\title{
Tratamiento concomitante de litio y metadona en un paciente bipolar: a propósito de un caso
}

\section{Concomitant lithium and methadone treatment in a bipolar patient: a case report}

Carlos Roncero*; Cristina lópez-Ortiz ${ }^{* *}$; Carmen Barral ${ }^{* * *}$; Nala SáeZ-Francàs ${ }^{* *}$; Marc Rovira ${ }^{* * *}$

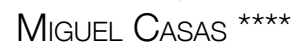

* Coordinador del CAS Vall d'Hebron. Servicio de Psiquiatría del Hospital Universitario Vall d'Hebrón. Profesor asociado de la Universitad Autónoma de Barcelona

** Servicio de Psiquiatría del Hospital Universitario Vall d'Hebrón.

*** CAS Vall d'Hebron. Servicio de Psiquiatría del Hospital Universitario Vall d'Hebrón.

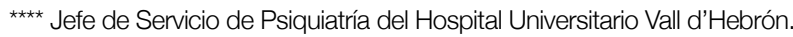
Catedrático de Psiquiatría de la Universidad Autónoma de Barcelona

Enviar correspondencia a:

Cristina López Ortiz, Hospital Universitario Vall d'Hebron; Senvicio de Psiquiatría, Escuela de enfermería $5^{\circ}$ planta; Passeig Vall d’hebron 119-129; código postal 08035; Barcelona. Correo electrónico crilopez@vhebron.net o croncero@vhebron.net recibido: octubre 2008 aceptado: noviembre 2008

\section{RESUMEN}

La patología dual requiere aproximaciones terapéuticas complejas para adecuar el tratamiento a la doble naturaleza del problema. Es importante conocer los efectos e interacciones medicamentosas que puedan acontecer para evaluar la sintomatología que refiere el paciente.

Dados los escasos datos sobre los efectos del tratamiento con litio y metadona, el objetivo de la exposición de este caso clínico es explicar las interacciones producidas entre metadona y carbonato de litio, en una paciente tratada con ambos fármacos, afecta de pluripatología médica y con dependencia de opiáceos y trastorno bipolar. Se exponen los diferentes efectos adversos más frecuentes de cada fármaco. Se describe de forma retrospectiva y prospectiva el manejo del tratamiento y las interacciones entre estos dos fármacos mediante la revisión de la historia clínica de la paciente y se detalla la correspondencia temporal entre las dosis de cada uno de ellos y la evolución. Tras un año de seguimiento, la paciente no ha sufrido interacciones en el tratamiento concomitante de litio y metadona.

El tratamiento en pacientes bipolares duales es complejo y debe ser realizado por equipos expertos.

Palabras clave: litio, metadona, trastorno bipolar, patología dual, tratamiento.

\section{ABSTRACT}

Dual diagnosis requires complex therapeutic approaches to adapt treatment to the dual nature of the problem. It is important to understand the drug effects and the drug interactions that may be observed during the integral treatment of these pathologies. Given the scarcity of data on the effects of lithium and methadone treatment, the aim of this clinical case report is to explain the interactions produced between methadone and lithium carbonate, in a female patient with medical multi-pathology, opioid-dependent and with comorbid bipolar disorder, who was treated with both drugs. The most common adverse effects for each drug are described. Drug interactions and temporal correspondence of the two drugs, as well as their dosages, are described through review of the retrospective chart and prospective follow-up of the patient. After oneyear of follow-up the patient had experienced no interactions in the concomitant lithium and methadone treatment.

It should be noted that treatment of bipolar patients with dual diagnosis is complex, and should only be carried out by experts.

Key words: lithium, methadone, bipolar disorder, dual diagnosis, treatment. 


\section{INTRODUCCIÓN}

L a prevalencia de trastornos psiquiátricos es 10 veces mayor en pacientes en Programa Mantenimiento con Metadona (PMM) en comparación con la población general' ${ }^{1}$. Existe gran variabilidad en las prevalencias de comorbilidad psiquiátrica en dependientes de opiáceos, debido a que las poblaciones estudiadas son muy heterogéneas ${ }^{2}{ }^{3}$. No obstante, entre los dependientes de opiáceos que acuden a tratamiento, los trastornos depresivos, de ansiedad y los trastornos de personalidad antisocial (TAP) y límite (TLP), son los diagnósticos más frecuentes ${ }^{3}$. En una población de 716 individuos en $P M M$, se describió que el 47\% presentaba algún trastorno psiquiátrico, siendo los más frecuentes el TAP (25.1\%) y la depresión mayor $(15.8 \%)^{4}$. En una muestra de 350 dependientes de opiáceos (42\% en PMM), los trastornos del eje I más frecuentes fueron los de ansiedad (43\%), seguidos de los trastornos afectivos $(32 \%)^{5}$, en otra de 132 pacientes en PMM los diagnósticos más frecuente fueron el trastorno de personalidad (51\%) y los trastornos afectivos (29.7\%). En 149 heroinómanos de fuera del circuito sanitario se describió una prevalencia de $33 \%$ de TAP y $26 \%$ de trastornos afecti$\operatorname{vos}^{7}$. En el conjunto de estudios el trastorno depresivo es el más frecuente y el que muestra mayor consistencia, aunque es difícil distinguir si los trastornos son primarios o inducidos $^{3}$. Por otra parte, el trastorno bipolar (TB) es el de mayor riesgo de comorbilidad con trastorno por uso de sustancias (TUS $^{8,9,10}$. La prevalencia de consumo de opiáceos no es de las más frecuentes y varia largo de la vida del 6-25\%10, 11 . La coexistencia entre un TUS y otro trastorno mental, implica que ambas patologías podrían compartir un marco anatómico y fisiológico común en el sistema nervioso central ${ }^{12}$, por ello el resultado de la presencia conjunta y de su mutua interacción, no provoca únicamente la conjunción clínica característica de ambos, sino la fusión sobre la que construir un modelo psicopatológico combinado ${ }^{12}$.

El litio es uno de los fármacos de elección en el tratamiento del TB y la metadona es el fármaco más ampliamente utilizado en el tratamiento de los dependientes de opiáceos ${ }^{1,4,5,6}$, por lo que se deberían conocer adecuadamente las propiedades y las interacciones de ambos fármacos en el tratamiento del TB con litio en pacientes que siguen un PMM. El litio es un catión monovalente, que se absorbe en el tracto gastrointestinal, se une mínimamente a proteínas, no sufre biotransformación y se elimina por vía renal al cabo de 24 horas $^{13,14}$. Presenta un estrecho margen terapéutico, que obliga a un control cuidadoso de sus concentraciones plasmáticas. Su excreción está controlada por factores osmóticos y está disminuida en la insuficiencia renal $^{15}$. Las concentraciones en el estado de equilibrio se alcanzan a los 4-5 dias ${ }^{16}$. Es eficaz a largo plazo para reducir la frecuencia de los episodios de alteración del estado de ánimo en los pacientes con trastorno bipolar I y $\mathrm{II}^{17}$. No se conoce exactamente su mecanismo de acción como estabilizador del estado de ánimo.

La metadona es el agonista opiáceo más empleado en el tratamiento de mantenimiento. Se absorbe en el tracto gastrointestinal. Se detecta en el plasma a los 30 minutos de la ingesta, llega a sus concentraciones máximas a las 4 horas. Tiene una biodisponibilidad media del 75\% ${ }^{18,19}$. El $90 \%$ se une a proteínas plasmáticas, es lipofílica. Sus concentraciones máximas en el cerebro se observan 1-2 horas, después de la administración subcutánea o intramuscular. Se metaboliza a nivel microsomal hepático por isoenzimas del citocromo P450, principalmente por CYP2D6, CYP1A2 y CYP3A4, sigue un proceso de $\mathrm{N}$-desmetilación y posterior ciclación. Los metabolitos principales son excretados en orina junto con pequeñas cantidades del compuesto no alterado. La velocidad del metabolismo de la metadona depende principalmente de la función del enzima CYP3A4 y su capacidad para inhibir el citocromo P450 2D6, que afecta el metabolismo de otros fármacos, por lo que presenta muchas interacciones. Su concentración alcanza el estado de equilibrio tras pocos días de administración diaria, siendo su fluctuación mínima. La semivida plasmática es de 22 horas aunque hay importantes diferencias según la edad ${ }^{19} \mathrm{y}$ la variabilidad genética asociada a la raza ${ }^{20}$.

\section{Interacción entre litio y metadona}

Existen diversos estudios experimentales en los que no se ha descrito un efecto negativo de la interacción litio/ opiáceos sino al contrario ${ }^{21}$. El litio puede alterar los efectos de los opiáceos sobre el sistema opioide ${ }^{22,23}$. Basándose en estudios experimentales, se propuso que el litio pudiera ser útil en clínica para el tratamiento de la adicción a opiáceos ${ }^{24,25}$, pues reduce significativamente la autoestimulación facilitada por morfina ${ }^{26}$, la auto-administración de morfina ${ }^{27}$ o la expresión del síndrome de abstinencia en animales dependientes de morfina, impidiendo el desarrollo de la dependencia ${ }^{28}$. No se conoce si las interacciones del litio se deben a acciones directas sobre el sistema opioide endógeno o a nivel subcelular ${ }^{21}$. No existen estudios en humanos sobre la eficacia de litio en pacientes dependientes a opiáceos. Por otra parte, se ha sugerido el efecto estabilizador del humor de la metadona en un paciente con TB y en tratamiento en $\mathrm{PMM}^{29}$. Se expone un caso clínico de una paciente con diagnóstico dual (TB y dependencia de opiáceos) cuyas características clínicas son idóneas para la observación de posibles interacciones adversas entre litio y metadona. Hay pocos trabajos que describan la evolución clínica y las interacciones farmacológicas en pacientes en PMM y TB. Se consideran las posibles interacciones presentadas por el resto de los psicofármacos pautados, como son la olanzapina y la gabapentina. En este sentido, se ha descrito buena tolerarancia del tratamiento concomitante con olanzapina y metadona y ausencia de interacciones farmacocinéticas entre ellos, no existiendo modificaciones de los niveles plasmáticos de metadona ${ }^{30,31}$. La gabapentina ha sido utilizada en procesos de desintoxicación de opiáceos ${ }^{32}$, y los escasos datos relacionados con efectos secundarios de la administaración concomitante de ambos fármacos, finalmente han sido relacionados exclusivamente con el uso de metadona $^{33}$. 


\section{MÉTODOS}

Se documenta la evolución del tratamiento con litio y metadona en una mujer de 45 años mediante el estudio de su historia clínica de forma retrospectiva y posterior seguimiento de un año prospectivo. Se identifica la dosificación de ambos medicamentos y de todo su tratamiento a lo largo del tiempo del estudio. Se registran los posibles efectos adversos derivados de la interacción entre litio y metadona habiéndose descartado de forma razonable otra etiología causante. Se conocen los efectos secundarios de ambos fármacos (Tabla 1) 13, 18,33, 34 de forma individual, pero la interacción entre ambos podría provocar un aumento de la intensidad y la frecuencia de los mismos. Además si se administran conjuntamente es posible la aparición de nuevos efectos no contemplados por separado.

Tabla 1. Efectos Adversos de litio y metadona.

\begin{tabular}{|l|l|}
\hline \multicolumn{1}{|c|}{ Litio } & \multicolumn{1}{c|}{ Metadona } \\
\hline Diarrea & Estreñimiento \\
Alteraciones cognitivas y menor & Náuseas y Vómitos \\
productividad asociativa & Anorexia \\
Coordinación motora & Hipersudoración \\
Alteración de habilidades motoras finas & Miosis \\
Neurotoxicidad & Sedación \\
Delirium & Depresión respiratoria y cardiovascular \\
Encefalopatia. & Linfocitosis \\
Temblor postural fino & Aumento de las concentración \\
Aumento de peso & plasmática de prolactina, albúmina y \\
Lesión tubular renal & globulinas. \\
Corea & Edemas \\
& Corea \\
\hline
\end{tabular}

\section{Historia clínica}

Antecedentes familiares: Hermano menor historia de TUS.

Antecedentes personales: Desarrollo psicomotor normal. Dificultad de atención en la escuela. Abandona los estudios primarios a los 15 años. Realiza actividad laboral irregular sobre todo en el campo de la hostelería. Cobra pensión no contributiva desde los 44 años. Divorciada a los 36 años, tiene 3 hijos a cargo del padre.

Antecedentes médicos: $\mathrm{VHC}+$ y $\mathrm{VHB}+$. Histerectomía por miomas uterinos a los 32 años. Exéresis de quiste ovárico derecho. Cirrosis hepática, desde los 44 años ha presentado descompensaciones relacionadas con abandono de medicación (ascitis, edemas maleolares y encefalopatía hepática).

\section{Antecedentes psiquiátricos toxicológicos:}

Tabaco: Inicio del consumo a los 12 años. Dosis acumulada de 33 paquetes/año. Consumo actual de 20 cigarrillos/día.

Alcohol: Inicio del consumo diario a los 14 años llegando hasta de 20 UBE / día a los 20 años. Se mantiene abstinente durante 8 años. Coincidiendo con separación matrimonial recaída en el consumo unos dos meses (10 UBE/dia). Abstinente en la actualidad.

Cocaína: Inicio a los 21 años, realizando un consumo diario vía intranasal de $0.5-1 \mathrm{~g}$./día. Periodo de abstinencia de unos 5 meses a los 32 años. Recaída y varios ingresos de desintoxicación, el último a los 44 años. Actualmente consumo puntual.

Cannabis: Consumo diario desde los 12 años hasta la actualidad de 1 unidad/día.

Hipnosedantes: Inicia consumo a los 44 años, aumentando hasta $6 \mathrm{mg}$ de Alprazolam y $20 \mathrm{mg}$ de Cloracepato al día, con importante afectación del nivel cognitivo y disminución del nivel de conciencia y sedación en relación a su hepatopatía de base.

Opiáceos: Inicia el consumo de heroína a los 15 años de forma esporádica, a los 22 años comienza a utilizar la vía intravenosa realizando consumo diario durante 2 años de 1/2-1 g/día vía intranasal. Ha presentado dos episodios de sobredosis. Se mantiene abstinente desde los 24 años. A los 43 años inicia consumo ilegal de metadona vinculándose meses después a la red de atención a drogodependientes, iniciándose el PMM.

Diagnóstico final: dependencia de opiáceos en tratamiento de mantenimiento con metadona, trastorno de dependencia de nicotina y abuso de cannabis y benzodiacepinas y trastorno por dependencia de cocaína y alcohol en remisión.

\section{Otros antecedentes psiquiátricos:}

Trastorno bipolar tipo I diagnosticado a los 19 años. Intento de defenestración a los 42 años, con ingreso en traumatología. Seguimiento ambulatorio inconstante. Inicio tratamiento con carbonato de litio $400 \mathrm{mg} /$ día a los 44 años (febrero del 2006), coincidiendo con un ingreso hospitalario para desintoxicación de cocaína. Previamente no realizaba tratamiento con estabilizadores del ánimo. Mala adherencia al tratamiento y seguimiento irregular en su Centro de Salud Mental de zona, desde septiembre del 2006.

Tratamiento (Tabla II): Carbonato de litio 400mg/dia; olanzapina $10 \mathrm{mg} /$ dia; gabapentina $1200 \mathrm{mg} /$ dia; metadona $35 \mathrm{mg} /$ dia; lactitol $30 \mathrm{~g} / \mathrm{dia}$; espirinolactona $100 \mathrm{mg} /$ dia; ácido fólico $5 \mathrm{mg} /$ día y fitomenadiona semanal.

La ausencia de dos controles de litemia se debe al cumplimiento irregular de los controles.

Tabla 2. Tratamiento psicofarmacológico.

\begin{tabular}{|l|l|l|l|l|l|l|}
\hline Año & \multicolumn{4}{|l}{2006} & \multicolumn{2}{l|}{2007} \\
\hline & Feb. & Ago. & Oct. & Nov. & Ene. & Feb. \\
\hline Litio (mg/dia) & 400 & 400 & 400 & 400 & 600 & 400 \\
\hline Litemia (meq/l) & 0.73 & 0.45 & 0.56 & 0.30 & -- & -- \\
\hline Metadona (mg/dia) & 35 & 45 & 35 & 35 & 30 & 25 \\
\hline Olanzapina (mg/dia) & 10 & 10 & 10 & 10 & 10 & 10 \\
\hline Gabapentina (mg/dia) & 900 & 900 & 1200 & 1200 & 1200 & 1200 \\
\hline
\end{tabular}




\section{RESULTADOS}

Durante el año de seguimiento la paciente sufrió 2 episodios de encefalopatía que se relacionaron temporalmente con el abandono del tratamiento laxante y el abuso de benzodiacepinas, sin asociación a cambios en las dosis de metadona o litio. En la primera descompensación de agosto del 2006, presentó ascitis, edemas maleolares y signos de encefalopatía hepática. La paciente explica haber abandonado su tratamiento laxante habitual 1 mes antes, el resto de los fármacos prescritos habían sido mantenidos con la misma dosis. La segunda descompensación se produce en diciembre presentando un cuadro similar.

Se detectaron litemias en niveles infraterapeúticas en dos controles, no relacionadas con los cambios en la dosificación de metadona sino con el incumplimiento farmacológico, motivo por el que se aumentó la dosis de litio a partir de noviembre, disminuyéndose al detectarse el incumplimiento. El tratamiento con olanzapina permaneció constante y se aumentó en $300 \mathrm{mg}$ la dosis de gabapentina. No presentó ninguna descompensación psicopatológica desde el inicio del tratamiento con litio. No se ha observado ningún efecto adverso concomitante consecuencia de los fármacos a estudio, ni tampoco parece existir una interacción en la farmacocinética de ambos. La paciente no ha sufrido interacciones en el tratamiento concomitante de litio y metadona.

\section{DISCUSIÓN}

El tratamiento de pacientes duales con múltiples patologías orgánicas es especialmente complicado, siendo necesario conocer y detectar las interacciones medicamentosas. Este informe trata de una paciente que acude tanto a la red de drogodependencias como a la de salud mental, un caso prototípico de paciente dual con irregularidad en el cumplimiento farmacológico. Los sujetos incluidos dentro de PMM presentan frecuentemente enfermedades hepáticas, con abuso de medicamentos prescritos y sustancias ilegales ${ }^{18}$, por lo que son una población de interés para el estudio de posibles interacciones. En este caso no se hallan interacciones ni efectos adversos asociados al litio, la metadona 0 a su asociación. Es destacable que la paciente no presenta descompensaciones psicopatológicas en el seguimiento a pesar de que el litio estaba en niveles infraterapeúticos, lo que podría sugerir el efecto estabilizador del humor de la metadona, descrito en otros pacientes ${ }^{29}$. Si se consideran los efectos anti-disfóricos de los opiáceos, se puede hipotetizar que la heroína es consumida para detener la disforia presente en los trastornos afectivos, lo que supondría un fenómeno de auto-medicación ${ }^{35}$. Casi todo el conocimiento acerca de los efectos de los opiáceos se deriva de la experiencia basada en la observación en toxicómanos, su uso en el tratamiento de los trastornos mentales no ha sido investigado, debido al riesgo de producir dependencia, aunque existen indicios de sus efectos antidisfóricos, ansiolíticos y antiagresivos ${ }^{36}$. Hay observaciones clínicas de pacientes bipolares tipo I en PMM en los que este fármaco podría actuar como estabilizador del ánimo $0^{29,37}$ y como agente antipsicótico ${ }^{12,38}$.

En pacientes bipolares, el abuso de sustancias es un predictor de peor respuesta tanto al litio como a cualquier tratamiento $0^{9,39}$. Los pacientes duales requieren dosis más altas de fármacos estabilizadores ${ }^{40}$, más tiempo hasta la remisión del episodio afectivo agudo, presentan más duración de los sintomas subsindrómicos de manía o depresión clínicamente significativos, en comparación con los no duales ${ }^{41}$, aunque podrian mostrar una mayor respuesta a los nuevos fármacos antiepilépticos que al litio ${ }^{39}$. Esto puede ser debido a que los pacientes con TUS usualmente presentan estados mixtos 0 ciclación rápida, que clásicamente muestran peor respuesta al litio ${ }^{42}$. Por todo lo expuesto, es evidente que el tratamiento idóneo para los pacientes bipolares en PMM no es conocido definitivamente, siendo necesario, como en el caso clínico presentado, el uso de combinaciones farmacológicas. Mientras no haya suficiente evidencia lo recomendable es seguir las guias clínicas basadas en consenso ${ }^{43}$. Al tratar a pacientes con patología dual es conveniente:

10 Actuar sobre el trastorno por dependencia de opiáceos mediante un programa de fármacos antagonistas y fármacos anticraving si la dependencia es leve, o iniciar un programa de mantenimiento con metadona si la dependencia es moderada o severa, o la abstinencia no es objetivo realista.

$2^{\circ}$ Conseguir el control del estado de ánimo con fármacos estabilizadores del estado de ánimo.

30 Seguir una estrategia de prevención de recaídas y detectar descompensaciones afectivas en las fases iniciales.

\section{CONCLUSIONES}

Los sujetos incluidos dentro de PMM son una población de especial interés en el estudio de interacciones medicamentosas, debido a que concurren en ellos una serie de factores que los predisponen a presentar mayor interacción (enfermedades concomitantes, ingesta de medicamentos no prescritos, incumplimiento...). Existen pocos datos clínicos y escasísima literatura científica en humanos que permitan preveer los efectos del tratamiento combinado de litio y metadona. Diversos estudios experimentales no parecen mostrar un posible efecto negativo de la interacción litio/opiáceos sino al contrario y hay observaciones clínicas de pacientes bipolares en PMM en los que la metadona actuaría como un estabilizador del ánimo y como antipsicótico ${ }^{12,29,38}$. En enfermos bipolares, el TUS comórbido, es un predictor de peor respuesta al tratamiento con litio y en general a cualquier tratamiento ${ }^{9,12}$. Se puede utilizar litio en pacientes bipolares en PMM si su nivel de colaboración permite los controles habituales de litemia. Dado el difícil manejo de estos pacientes, se debería plantear la creación de programas específicos de abordaje de pacientes con patologia dual para evitar posibles recaídas favorecidas por la descoordinación en el complejo tratamiento de estos pacientes. 


\section{REFERENCIAS}

1. Callaly T, Trauer T, Munro L, Whelan G. Prevalence of psychiatric disorder in a methadone maintenance population. Aust N Z J Psychiatry 2002; 36: 426-427.

2. Torrens M, Martin-Santos $R$, Samet S. Importance of clinical diagnoses for comorbidity studies in substance use disorders. Neurotox Res 2006; 10: 253-261.

3. Mateu G, Astals M, Torrens M. Comorbilidad psiquiátrica y trastorno por dependencia de opiáceos: del diagnóstico al tratamiento. Adicciones 2005; 17 Supl 2: 111-121.

4. Brooner RK, King VL, Kidorf $M$, Schmidt CW, Bigelow GE. Psychiatric and substance use comorbidity among treatment seeking opioid abusers. Arch Gen Psychiatry 1997; 54: 71-80.

5. Krausz M, Verthein U, Degkwitz P. Prevalence of psychiatric disorders in opiate dependent patients in contact with the drug treatment system. Nervenarzt 1998; 69: 557-567.

6. Fernández Miranda JJ, González Garcia-Portilla M, Sáiz Martínez P, Gutiérrez Cienfuegos E, Bobes García J. Influencia de los trastornos psiquiátricos en la efectividad de un programa de mantenimiento prolongado con metadona. Actas Esp Psiquiatr 2001; 29: 228-232.

7. Rodríguez-Llera MC, Domingo-Salvany A, Brugal MT, Silva TC, Sánchez-Niubó A, Torrens M, ITINERE Investigators. Psychiatric comorbidity in young heroin users. Drug Alcohol Depend 2006; 84: 48-55.

8. Weiss RD, Kolodziej M, Griffin ML, Najavits LM, Jacobson LM, Greenfield SF. Substance use and perceived symptom improvement among patients with bipolar disorder and substance dependence. J Affect Disord 2004; 79: 279-283.

9. Brady KT, Sonne SC. The relationship between substance abuse and bipolar disorder. J Clin Psychiatry 1995; 56 Supl 3: 19-24.

10. Regier DA, Farmer ME, Rae DS, Locke BZ, Keith SJ, Judd LL et al. Comorbidity of mental disorders with alcohol and other drug abuse. Results from the Epidemiologic Catchment Area (ECA) Study. JAMA 1990; 264: 2511-8.

11. Brown ES, Suppes T, Adinoff B, Thomas NR. Drug abuse and bipolar disorder: comorbidity or misdiagnosis? J Affect Disord 2001; 65: 105-115.

12. Maremmani I, Perugi G, Pacini M, Akiskal Hs. Toward a unitary perspective on the bipolar spectrum and substance abuse: opiate addiction as a paradigm. J Affect Disord 2006; 93: 1-12.

13. Freeman MP, Wiegand $C$, Gelenberg AJ. Litio. En: Schatzberg $A F$, Nemerof $C B$, Editores. Tratado de psicofarmacología. 13 ed. Barcelona: Masson; 2006. p. 597-617.

14. Baldessarini RJ, Tarazi FI, Editors. Drugs and the treatment of psychiatric disorders: psychosis and mania. Goodman and Gilman's. The Pharmacological Basis of Therapeutics, 10 Ed. New York: McGraw-Hill, 2001. p. 485-520.

15. Birch NJ, Hullin RP. Lithium and the kidney. Br Med J 1980; 280: 1148-9.

16. Keck PE Jr, McElroy SL. Clinical pharmacodynamics and pharmacokinetics of antimanic and mood-stabilizing medications. J Clin Psychiatry 2002; 63 Supl 4: 3-11.

17. Tondo L, Hennen J, Baldessarini RJ. Lower suicide risk with long-term lithium treatment in major affective illness: a metaanalysis. Acta Psychiatr Scand 2001; 104: 163-72.
18. Colom J, Duro P, Editores. Tractament de manteniment amb metadona. Manual de pràctica clínica. Barcelona: Generalitat de Catalunya. Departament de Salut; 2005.

19. Vazquez $V$, Gury $C$, Laqueille $X$. Méthadone: de la pharmacocinétique à la pharmacologie clinique. Encéphale 2006; 32 (4 Pt 1): 478-86.

20. Eap CB, Broly F, Mino A, Hämmig R, Déglon JJ, Uehlinger $C_{\text {, et }}$ al. Cytochrome P450 2D6 genotype and methadone steady-state concentrations. J Clin Psychopharmacol 2001; 21: 229-34.

21. Moreno Brea MR, Rojas Corrales O, Gibert-Rahola J, Mico JA. Interacciones medicamentosas de metadona con fármacos activos del SNC. Actas Esp Psiquiatr 1999; 27: 103-10.

22. Mannisto PT, Saarnivaara L. Effect of lithium on the analgesia caused by morphine and two antidepressants in mice. Pharmacology 1972; 8: 329-35.

23. Staunton DA, Deyo SN, Shoemaker WJ, Ettenberg A, Bloom FE. Effects of chronic lithium on enkephalin systems and pain responsiveness. Life Sci 1982; 31: 1837-40.

24. Flemenbaum $A$, Cronson AJ, Weddige RL. Lithium in opiate abuse: a theoretical approach. Comprehensive Psychiatry 1979; 20: $91-9$.

25. Abrahamson JR. Use of lithium to control drug abuse. Am J Psychiatry 1983; 140: 1256.

26. Liebman JM, Segal DS. Lithium differentially antagonises selfstimulation facilitated by morphine and (+)-amphetamine. Nature 1976; 260: 161-3.

27. Tomkiewicz M, Steinberg $\mathrm{H}$. Lithium treatment reduces morphine self-administration in addict rats. Nature $1974 ;$ 252: 227-9.

28. Dehpour AR, Farsam $H$, Azizabadi-Farahani M. Inhibition of the morphine withdrawal syndrome and the development of physical dependence by lithium in mice. Neuropharmacology 1994; 34: 115-21.

29. Pani PP, Agus A, Gessa GL. Methadone as a mood stabilizer. Heroin Add \& Rel Clin Probl 1999; 1: 43-44.

30. Baño MD, Micó JA, Agujetas M, López ML, Guillén JL. Eficacia del antipsicótico olanzapina en el tratamiento del abuso de cocaína en pacientes en mantenimiento con metadona. Interacción en los niveles plasmáticos. Actas Esp Psiquiatr 2001; 29: 215-20.

31. Gerra G, Di Petta G, D'Amore A, lannotta P, Bardicchia F, Falorni $F_{1}$ et al. Combination of olanzapine with opioid-agonists in the treatment of heroin-addicted patients affected by comorbid schizophrenia spectrum disorders. Clin Neuropharmacol. 2007; 30: 127-35.

32. Martinez-Raga J, Sabater A, Perez-Galvez B, Castellano M, Cervera $G$. Add-on gabapentin in the treatment of opiate withdrawal. Prog Neuropsychopharmacol Biol Psychiatry 2004; 28: 599-601.

33. Kharlamb $V$, Kourlas $H$. Edema in a patient receiving methadone for chronic low back pain. Am J Health Syst Pharm 2007; 64: 2557-60.

34. Cardoso F. Chorea: non-genetic causes. Curr Opin Neurol 2004; 17: 433-6.

35. Khantzian EJ. The self-medication hypothesis of addictive disorders: focus on heroin and cocaine dependence. Am J Psychiatry 1985; 142: 1259-64.

36. Verebey K, Kogan MJ, Mule SJ. Phencyclidine-induced stereotype in rats: effects of methadone, apomorphine, and naloxone. Psychopharmacology (Berl) 1981; 75: 44-7.

37. Maremmani I, Pacini M, Perugi G, Deltito J, Akiskal H. Cocaine abuse and the bipolar spectrum in 1090 heroin addicts: clinical 
observations and a proposed pathophysiologic model. J Affect Disord 2008; 106: 55-61.

38. Levinson I, Galynker II, Rosenthal RN. Methadone withdrawal psychosis. J Clin Psychiatry 1995; 56: 73-6.

39. Goldberg JF, Garno JL, Leon AC, Kocsis JH, Portera L. A history of substance abuse complicates remission from acute mania in bipolar disorder. J Clin Psychiatry 1999; 60: 733-40.

40. Maremmani I, Zolesi O, Aglietti M, Marini G, Tagliamonte A, Shinderman $M$, et al. Methadone dose and retention during treatment of heroin addicts with Axis I psychiatric comorbidity. J Addict Dis 2000; 19: 29-41.

41. Gaudiano BA, Uebelacker LA, Miller IW. Impact of remitted substance use disorders on the future course of bipolar I disorder: Findings from a clinical trial. Psychiatry Res 2008; 160: 63-71.

42. Grunze $H$, Amann B, Dittmann S, Walden J. Clinical relevance and treatment possibilities of bipolar rapid cycling. Neuropsychobiology 2002; 45 Supl 1: 20-6.

43. Casas M, Franco MD, Goikoelea JM, Jiménez-Arriero MA, Martínez-Raga J, Roncero C, et al. Trastorno bipolar asociado al uso de sustancias adictivas (patología dual). Revisión sistemática de la evidencia científica y consenso entre profesionales expertos. Actas Esp Psiquiatr 2008; 36: 350-61. 